\title{
Inspection of Short-Time Resting-State Electroencephalogram Functional Networks in Alzheimer's Disease
}

\author{
Javier Escudero ${ }^{1, \&,}$, Member, IEEE, Keith Smith ${ }^{1,2, \&}$, Student member, IEEE, Hamed Azami ${ }^{1}$, Student \\ member, IEEE, and Daniel Abásolo ${ }^{3}$, Member, IEEE
}

\begin{abstract}
Functional connectivity has proven useful to characterise electroencephalogram (EEG) activity in Alzheimer's disease (AD). However, most current functional connectivity analyses have been static, disregarding any potential variability of the connectivity with time. In this pilot study, we compute short-time resting state EEG functional connectivity based on the imaginary part of coherency for 12 AD patients and 11 controls. We derive binary unweighted graphs using the cluster-span threshold, an objective binary threshold. For each short-time binary graph, we calculate its local clustering coefficient $\left(C_{l o c}\right)$, degree $(K)$, and efficiency $(E)$. The distribution of these graph metrics for each participant is then characterised with four statistical moments: mean, variance, skewness, and kurtosis. The results show significant differences between groups in the mean of $K$ and $E$, and the kurtosis of $C_{l o c}$ and $K$. Although not significant when considered alone, the skewness of $C_{l o c}$ is the most frequently selected feature for the discrimination of subject groups. These results suggest that the variability of EEG functional connectivity may convey useful information about AD.
\end{abstract}

\section{INTRODUCTION}

Alzheimer's disease (AD) is the most common type of dementia in Western societies [1]. The criteria for AD diagnosis have recently been revised by different groups, leading to recommendations for harmonisation that highlight the potential role biomarkers could play in diagnosis [2]. However, these biomarkers are invasive, expensive and/or confined to the hospital setting [2]. The process of diagnosis still remains complex and its accuracy is relatively low. Hence, we need better tools to help clinicians in the detection of early $\mathrm{AD}$ in an affordable, non-invasive, and portable way.

The electroencephalogram (EEG) is a direct and noninvasive multichannel recording of the electric potentials generated by neural activity. The EEG reflects the transient temporal fluctuations of brain activity thanks to its high temporal resolution, which is orders of magnitude better than other techniques (e.g, functional magnetic resonance imaging, fMRI). Furthermore, the EEG is portable and

This work was partially supported by the Engineering and Physical Sciences Research Council (EPSRC) via a DTP studentship to K. Smith and the research project EP/N014421/1 to J. Escudero.

\& denotes equal contribution. * indicates corresponding author.

${ }^{1}$ J. Escudero, K. Smith, and H. Azami are with the Institute for Digital Communications, School of Engineering, University of Edinburgh, King's Buildings, Edinburgh EH9 3FB. (javier.escudero@ed.ac.uk).

${ }^{2} \mathrm{~K}$. Smith is also with the Alzheimer Scotland Dementia Research Centre, School of Philosophy, Psychology and Language Sciences, University of Edinburgh 7 George Square, Edinburgh, EH8 9JZ.

${ }^{3}$ D. Abásolo is with the Centre for Biomedical Engineering, Department of Mechanical Engineering Sciences, Faculty of Engineering and Physical Sciences, University of Surrey, Guildford GU2 7XH, UK affordable [3]. Due to these advantages, numerous EEG features have been considered to characterise AD [4]. Among them, analyses based on EEG functional connectivity (the set of statistical dependencies between spatially distinct signals) have recently gained attention [5]. Functional connectivity is useful to evaluate synchronisation and to understand the organised behaviour of brain networks, including how they change in conditions such as AD, epilepsy, schizophrenia and, even, ageing, to name a few [5]-[8].

Despite the understanding of brain activity facilitated by classical functional connectivity, the findings are inherently limited by the fact that most tools disregard the potential presence of dynamic changes in the connectivity patterns. They only provide a "static" (i.e., time-independent) view of functional networks for each person. This is in contrast with the dynamic behaviour of brain activity. Indeed, one of its hallmarks is the presence of spontaneous fluctuations that lead to network dynamics spanning short and long temporal scales [9]. This represents a paradigm change in the characterisation of brain function, which is further supported by computational models showing that the existence of dynamic fluctuations in brain functional connectivity is a natural consequence of large-scale dynamics [9].

Thus, it comes as no surprise that several groups have tried to study the dynamics of functional brain networks in fMRI [10]-[12], and resting-state electrophysiological signals [13]-[15]. The results reveal new potential signatures of diseases such as multiple sclerosis [10] and Parkinson's disease [12], thus opening a potentially new way to detect and monitor brain diseases over and beyond current techniques. This is particularly the case for EEG activity due to its high temporal resolution [3].

In that vein, this pilot study represents a first step towards the assessment of the variability of short-time resting-state EEG dynamical connectivity for the characterisation of AD. Instead of charactering each participant with a single, static, functional connectivity matrix, we will consider graph features computed from short-time connectivity matrices and we will then evaluate their distribution for each subject. Our choice of $\mathrm{AD}$ is supported by the fact that this condition damages brain connections [5], [8] and preliminary evidence showed that it also affects the temporal evolution of brain activity [16], [17]. Hence, we hypothesise that the analysis of the temporal variability of short-time EEG connectivity could constitute a new way to monitor AD.

\section{MATERIALS}

\section{A. Subjects}

Resting state EEG activity was acquired from $12 \mathrm{AD}$ 
patients and 11 age-matched healthy control subjects. The patients -7 women; age $=72.8 \pm 8.0$ years, mean \pm standard deviation (SD) - were recruited from the Alzheimer's Patient's Relatives Association of Valladolid (AFAVA), Spain. All patients met the criteria for probable AD according to NINCDS-ADRDA guidelines and they underwent clinical evaluations including clinical history, neurological and physical examinations, and brain scans. Their average minimental state examination (MMSE) was $13.3 \pm 5.6$ points (mean \pm SD). However, five patients had an MMSE below 12 points, indicating severe dementia. Two patients were taking lorapezam, which may enhance $\beta$ activity with therapeutic doses but no prominent rapid rhythms were observed in the visual inspection of their EEGs. No other patients were taking medication that could affect the EEG. The 11 control subjects -4 women; $72.8 \pm 6.1$ years \pm SD did not have any past or present mental disorder. They all scored 30 on the MMSE. The local ethics committee approved the study. All control subjects and patients' caregivers gave their informed consent for participation [18].

\section{B. EEG Recording}

The EEGs were recorded with Profile Study Room 2.3.411 EEG equipment (Oxford Instruments). The electrodes were placed following the international 10/20 system at locations Fp1, Fp2, F3, F4, F7, F8, C3, C4, T3, T4, T5, T6, P3, P4, O1, and O2. At least five minutes of data were recorded for each volunteer. Subjects were asked to remain in a relaxed awakened state with eyes closed during the recording process. Sampling was performed at $256 \mathrm{~Hz}$ with 12-bit A-to-D precision. Recordings were visually inspected by a specialist physician who selected epochs of minimally artefactual activity of $5 \mathrm{~s}$ (1280 sample points) from the data for further analysis. The average number of $5 \mathrm{~s}$ epochs per electrode per subject was $28.8 \pm 15.5$ (mean \pm $\mathrm{SD})$. The recordings were finally exported to ASCII format to a computer for off-line analysis [18].

\section{METHODS}

\section{A. Preprocessing}

The EEG signals were pre-processed with FieldTrip [19]. For each epoch, the 16 channels were re-referenced to the grand average [7]. As we are interested in inspecting shorttime features of the EEG functional connectivity, we further split each $5 \mathrm{~s}$ epoch into 'trials' of $1 \mathrm{~s}$ without overlap and padded with zeros to a length of $2 \mathrm{~s}$ to achieve a $0.5 \mathrm{~Hz}$ frequency resolution. We then used the muli-taper method in Fieldtrip with a Hanning window to compute the frequency cross-spectrum for all pairs of EEG channels [19]. Finally, we computed the imaginary part of coherence to assess the statistical dependencies between EEG channels between $0.5 \mathrm{~Hz}$ and $40 \mathrm{~Hz}$. The imaginary part of coherency is based on traditional coherency but the influence of volume conduction is minimised by only considering the imaginary part of the result [7]. We computed one connectivity matrix per short-time $1 \mathrm{~s}$ trial.

\section{B. Network binarisation}

We use Graph Theory [5], [20] concepts to characterise the short-time EEG functional connectivity captured in each 1 s connectivity matrix. Consider $G=(V, E, \mathbf{A})$ an undirected graph where $V$ and $E_{\text {denote }}$ the set of vertices and edges, respectively, and $\mathbf{A}$ is a weighted adjacency matrix obtained as the absolute value of the imaginary part of coherency. We then consider binary undirected graphs due to their simplicity and widespread use in brain connectivity analyses [8], [21]. This requires setting a threshold such that connections with weights larger than it are kept in the binary network and links with weights smaller than the threshold are discarded [7], [21], [22]. To avoid subjectivity in its selection, we resort to the recently proposed cluster-span threshold (CST) [22], which computes an extensive list of proportional thresholds and selects the one for which the proportion of open triples to closed triples is balanced in the graph. This balance occurs exactly where the global clustering coefficient is equal to 0.5 . For additional details, see [22].

\section{Network metrics}

We characterise each binary network with three straightforward and commonly used metrics: mean local clustering coefficient $\left(C_{l o c}\right)$, degree centrality $(K)$, and efficiency $(E)$ [20], [21].

For node $i$, with $k$ neighbours (nodes sharing edges with $i$ ), the local clustering coefficient of $i$ is the percentage of pairs of neighbours of $i$ which are themselves neighbours. The mean local clustering coefficient of a graph $\left(C_{l o c}\right)$ is thus the mean of the local clustering coefficients of all nodes. The degree of a given node is the number of neighbours of the node - i.e., the number of nodes with which the given node shares an edge. The degree centrality of a graph $(K)$ is thus the mean of the degrees of all the nodes. The efficiency of a graph $(E)$ is the mean of the values of the inverse of shortest path lengths between each pair of nodes, where a path between two nodes is a sequence of non-repeating edges connecting one to the other and its length is the size of that sequence. Thus, it is something akin to a normalised characteristic path length for the graph. Large values tell us that the connections of the network are highly integrated. Low values indicate segregated connections [20], [21].

\section{Analysis and comparison between groups}

To characterise the potential variability in the short-time EEG connectivity due to $\mathrm{AD}$, we gather the results of each of the network features $\left(C_{l o c}, K\right.$, and $\left.E\right)$ computed from the $1 \mathrm{~s}$ signal trials for each subject. For each subject, these values can be seen as a distribution, which we then characterise computing its statistical moments: mean, variance, skewness, and kurtosis. Therefore, for each participant, we consider four statistical moments computed for three network metrics.

We use a Student's $t$-test to assess the level of differences between AD patients and controls. We hypothesise that any potential effect of $\mathrm{AD}$ in the short-time behaviour and variability of the EEG functional connectivity between groups would be reflected in the appearance of differences between groups. We also assess the complementarity of the moments of the network metrics by applying a forward sequential feature selection procedure with linear discriminant analysis as a classifier (without any limit on the maximum number of features that could be selected). The objective is to find the combination of features that have predictive power about diagnosis. We repeat this procedure 100 times to different stratified five-fold cross-validation splits of the subjects. 


\section{RESULTS AND DISCUSSION}

For each participant, we obtained a distribution of graph metrics $\left(C_{l o c}, K\right.$, and $\left.E\right)$ computed from short-time intervals of $1 \mathrm{~s}$. Each subject' distribution was then characterised with four statistical moments, and Student's $t$-tests were used to assess the level of differences between controls and $\mathrm{AD}$ patients. The corresponding $p$-values appear in Table I. The results suggest the presence of statistical differences in the distribution of graph metrics due to $\mathrm{AD}$. The grand average values of the mean of $K$ were $0.484 \pm 0.005$ and $0.493 \pm$ 0.012 for $\mathrm{AD}$ patients and controls, respectively (all results given as mean $\pm \mathrm{SD}$.) For $E$, the grand averages were $0.736 \pm$ 0.004 in $\mathrm{AD}$ and $0.742 \pm 0.008$ in controls. In static EEG connectivity analyses, $\mathrm{AD}$ has been associated with lower normalised characteristic path length and more random networks [5], [8]. We have found slightly lower values of $K$ and, notably, $E$ in AD. These findings must be treated with caution as the choice of connectivity metric (imaginary part of coherency in our case) may affect the result [5], [8]. However, they might suggest that different perspectives on the effect of AD on EEG networks could be found depending on whether one analyses just one single static network or the whole set of short-time ones.

Of note is that the kurtoses of the distribution of $C_{l o c}$ and $K$ seem to differ slightly for AD patients and controls. This is indicative of the subject groups having distributions of graph metrics with slightly different shape. Kurtosis is a measure of the "peakness" of the probability density function of a real random variable. For $C_{l o c}$, the grand average results of kurtosis for AD patients and controls were 5.247 \pm 1.504 and $3.910 \pm 1.240$, in that order. In the case of $K$, the AD patients had a grand average of 5.681 \pm 2.360 , whereas the corresponding value for the control subjects was $3.739 \pm$ 1.632. These results indicate that different subjects from different groups may be characterised with different distributions, with those of $\mathrm{AD}$ patients being dominated more frequently by single values of this metrics (higher kurtosis), thus potentially suggesting a reduced repertoire of connectivity configurations during resting-state in AD. For illustration purposes, Fig. 1 shows the distributions of the values of $C_{l o c}, K$, and $E$ computed from the short-time graphs for one control subject and one $\mathrm{AD}$ patient.

Finally, we assessed the potential complementarity of the moments of the graph metrics for the separation of $\mathrm{AD}$ patients and controls. We ran 100 independent repetitions of a sequential feature selection process where the ability of the features to separate both groups was evaluated with a linear discriminant analysis. Fig. 2 shows the relative frequency of selection for each feature (each moment of each graph metric). Features such as the mean value of $K$ or $E$ ('me $K$ ' and ' $m e E$ ' in Fig. 2) are selected in approximately $10 \%$ to

TABLE I. $\quad P$-VALUES FOR THE DIFFERENCES BETWEEN AD PATIENTS AND CONTROLS IN THE MOMENTS OF GRAPH METRICS COMPUTED FOR SHORT-TIME EEG CONNECTIVITY MATRICES BINARISED WITH THE CST.

\begin{tabular}{|c|c|c|c|c|}
\hline \multirow{2}{*}{$\begin{array}{c}\text { Network } \\
\text { metric }\end{array}$} & \multicolumn{4}{|c|}{ Statistical moment } \\
\cline { 2 - 5 } & Mean & Variance & Skewness & Kurtosis \\
\hline$C_{\text {loc }}$ & 0.4401 & 0.9583 & 0.1401 & 0.0310 \\
\hline$K$ & 0.0204 & 0.2992 & 0.1173 & 0.0336 \\
\hline$E$ & 0.0225 & 0.0934 & 0.0499 & 0.0573 \\
\hline
\end{tabular}

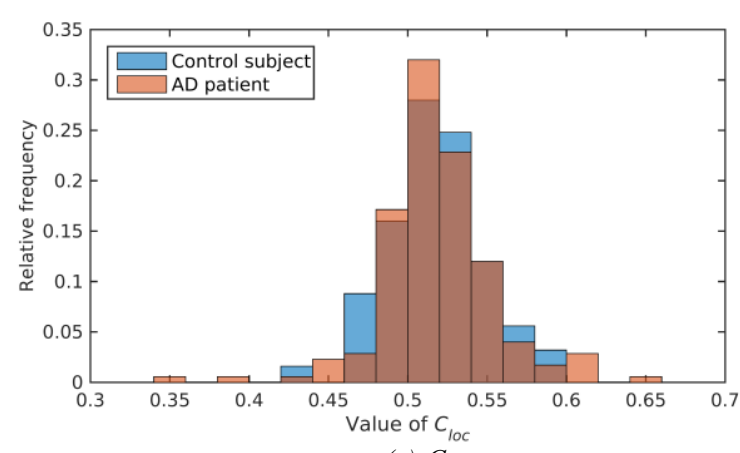

(a) $C_{l o c}$

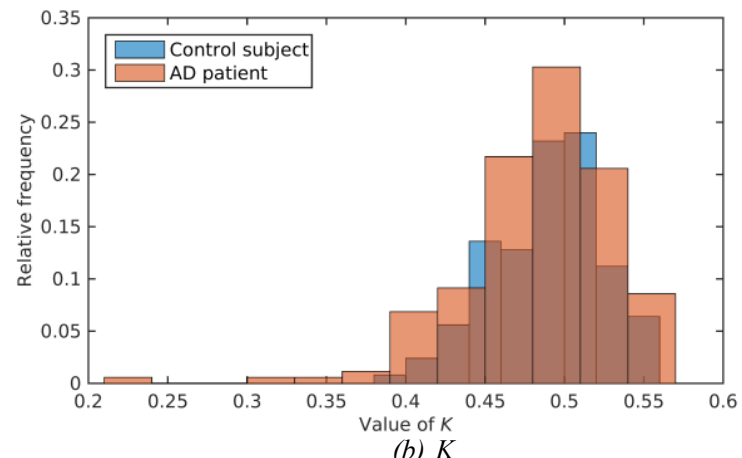

(b) $K$

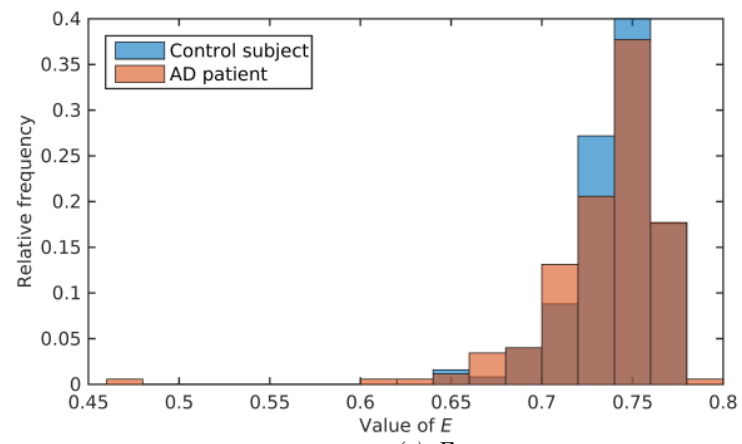

(c) $E$

Figure 1. Example of the distributions of values of graph metrics for one control subject (light blue) and one AD patient (light orange).

$20 \%$ of the independent runs of the feature selection. However, the most commonly selected feature is the level of skewness of $C_{l o c}$ ('skC' in Fig. 2, selected in $>60 \%$ of the runs). This indicates that various descriptors of the graph metric distributions can have complementary information to each other features, even if they do not convey statistically significant differences between groups on their own. This is shown in Fig. 3 with a scatter plot with the values of the two most frequently used features (skewness of $C_{l o c}$ and mean of $K$ ) for both subject groups. The consideration of short-time functional connectivity information could help to reveal features that would be otherwise not available in traditional analyses of "static" connectivity information. This agrees with previous research such as [10], [12].

\section{CONCLUSION}

We have presented pilot results on the inspection of shorttime resting state EEG functional connectivity in AD. Our results were limited by the small sample size and low-density of the EEG recordings but they suggested that the evaluation of functional connectivity in shorter temporal intervals than 


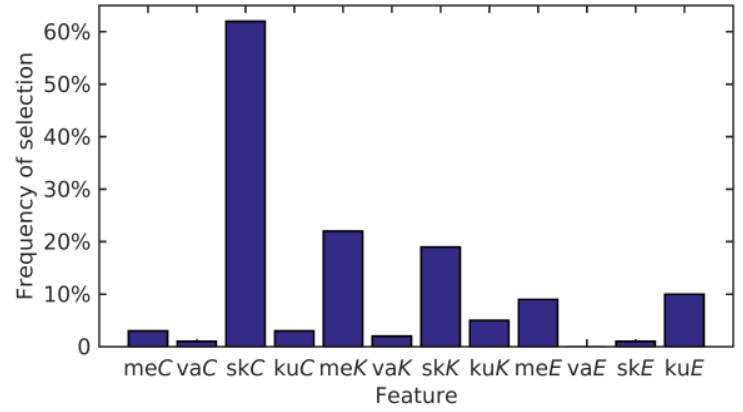

Figure 2. Proportion of times that a particular feature was selected. $C_{l o c}$ is abbreviated in the figure as ' $C$ '. 'mean', 'variance', 'skewness', and 'kurtosis' are shown as 'me', 'va', 'sk', and 'ku', respectively.

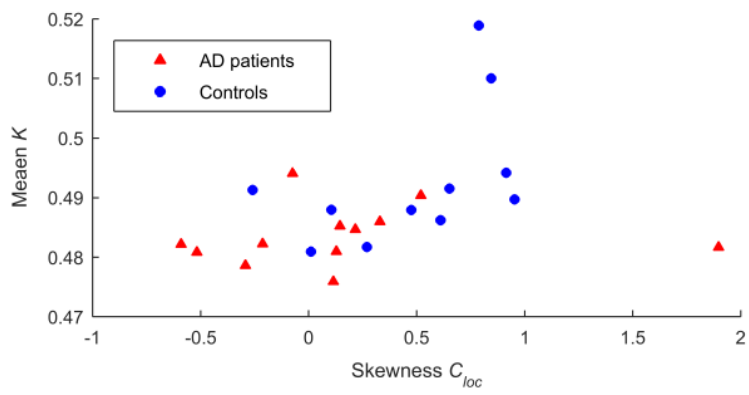

Figure 3. Scatter plot of the values of the skewness of $C_{l o c}$ (x axis) and mean of $K$ (y axis) for AD patients (red triangles) and control subjects (blue circles).

traditionally performed may lead to new information about how disease affects the brain activity. This provides additional support for the study of dynamical functional connectivity [9], a topic that could become even more relevant in EEG studies than in fMRI ones due to the excellent temporal resolution of the former [3]. Our future research will aim at overcoming technical issues such as the choice of fixed windows lengths ( $1 \mathrm{~s}$ in this study) and, more importantly, modelling the temporal dependencies spanning multiple temporal scales, using concepts from tensor factorisations [23] and temporal networks [24].

\section{REFERENCES}

[1] Alzheimer's Disease International, 'World Alzheimer Report: Policy Brief for Heads of Government - The Global Impact of Dementia 2013-2050', 2013.

[2] J. C. Morris, K. Blennow, L. Froelich, A. Nordberg, H. Soininen, G. Waldemar, L.-O. Wahlund, and B. Dubois, 'Harmonized diagnostic criteria for Alzheimer's disease: recommendations', J. Intern. Med., vol. 275, no. 3, pp. 204-213, 2014.

[3] F. Lopes da Silva, 'EEG and MEG: Relevance to Neuroscience', Neuron, vol. 80, no. 5, pp. 1112-1128, Dec. 2013.

[4] N. K. Al-Qazzaz, S. H. B. M. Ali, S. A. Ahmad, K. Chellappan, M. S. Islam, and J. Escudero, 'Role of EEG as Biomarker in the Early Detection and Classification of Dementia', Sci. World J., vol. 2014, p. e906038, Jun. 2014.

[5] C. J. Stam, 'Modern network science of neurological disorders', Nat. Rev. Neurosci., vol. 15, no. 10, pp. 683-695, Oct. 2014.

[6] J. Escudero, S. Sanei, D. Jarchi, D. Abásolo, and R. Hornero, 'Regional coherence evaluation in mild cognitive impairment and Alzheimer's disease based on adaptively extracted magnetoencephalogram rhythms', Physiol. Meas., vol. 32, no. 8, pp. 1163-1180, Aug. 2011.

[7] E. van Diessen, T. Numan, E. van Dellen, A. W. van der Kooi, M. Boersma, D. Hofman, R. van Lutterveld, B. W. van Dijk, E. C. W. van Straaten, A. Hillebrand, and C. J. Stam, 'Opportunities and methodological challenges in EEG and MEG resting state functional brain network research', Clin. Neurophysiol., vol. 126, no. 8, pp. 1468-1481, Aug. 2015

[8] B. M. Tijms, A. M. Wink, W. de Haan, W. M. van der Flier, C. J. Stam, P. Scheltens, and F. Barkhof, 'Alzheimer's disease: connecting findings from graph theoretical studies of brain networks', Neurobiol. Aging, vol. 34, no. 8, pp. 2023-2036, Aug. 2013.

[9] V. D. Calhoun, R. Miller, G. Pearlson, and T. Adalı, 'The Chronnectome: Time-Varying Connectivity Networks as the Next Frontier in fMRI Data Discovery', Neuron, vol. 84, no. 2, pp. 262274, Oct. 2014

[10] N. Leonardi, J. Richiardi, M. Gschwind, S. Simioni, J.-M. Annoni, M Schluep, P. Vuilleumier, and D. Van De Ville, 'Principal components of functional connectivity: A new approach to study dynamic brain connectivity during rest', NeuroImage, vol. 83, pp. 937-950, Dec. 2013.

[11] E. A. Allen, E. Damaraju, S. M. Plis, E. B. Erhardt, T. Eichele, and V. D. Calhoun, 'Tracking Whole-Brain Connectivity Dynamics in the Resting State', Cereb. Cortex, vol. 24, no. 3, pp. 663-676, Jan. 2014.

[12] T. M. Madhyastha, M. K. Askren, P. Boord, and T. J. Grabowski, 'Dynamic connectivity at rest predicts attention task performance', Brain Connect., vol. 5, no. 1, pp. 45-59, Feb. 2015.

[13] S. Mehrkanoon, M. Breakspear, and T. W. Boonstra, 'LowDimensional Dynamics of Resting-State Cortical Activity', Brain Topogr., vol. 27, no. 3, pp. 338-352, May 2014.

[14] N. Dhulekar, B. Oztan, B. Yener, H. O. Bingol, G. Irim, B. Aktekin, and C. Aykut-Bingöl, 'Graph-theoretic Analysis of Epileptic Seizures on Scalp EEG Recordings', in Proc. 5th ACM Conf. Bioinf., Comp. Biol., Health Inf., New York, NY, USA, 2014, pp. 155-163.

[15] M. J. Brookes, G. C. O’Neill, E. L. Hall, M. W. Woolrich, A. Baker, S. Palazzo Corner, S. E. Robson, P. G. Morris, and G. R. Barnes, 'Measuring temporal, spectral and spatial changes in electrophysiological brain network connectivity', NeuroImage, vol. 91, pp. 282-299, May 2014.

[16] J. Escudero, A. Anastasiou, and A. Fernández, 'Post-Processing for Spectral Coherence of Magnetoencephalogram Background Activity: Application to Alzheimer's Disease', in Proc. 36th Ann. Int. Conf. IEEE Eng. Med. Biol., Chicago, US, 2014, pp. 6345-6348.

[17] J. Poza, R. Hornero, J. Escudero, A. Fernández, and C. Gomez, 'Analysis of spontaneous MEG activity in Alzheimer's disease using time-frequency parameters', in Proc. 30th Ann. Int. Conf. IEEE Eng. Med. Biol. Soc., 2008, pp. 5712-5715.

[18] J. Escudero, D. Abasolo, R. Hornero, P. Espino, and M. Lopez, 'Analysis of electroencephalograms in Alzheimer's disease patients with multiscale entropy', Physiol. Meas., vol. 27, no. 11, pp. 1091$1106,2006$.

[19] R. Oostenveld, P. Fries, E. Maris, and J.-M. Schoffelen, 'FieldTrip: Open Source Software for Advanced Analysis of MEG, EEG, and Invasive Electrophysiological Data', Comput. Intell. Neurosci., vol. 2011, Dec. 2010.

[20] E. Bullmore and O. Sporns, 'Complex brain networks: graph theoretical analysis of structural and functional systems', Nat. Rev. Neurosci., vol. 10, no. 3, pp. 186-198, Mar. 2009.

[21] F. D. V. Fallani, J. Richiardi, M. Chavez, and S. Achard, 'Graph analysis of functional brain networks: practical issues in translational neuroscience', Philos. Trans. R. Soc. B Biol. Sci., vol. 369, no. 1653, p. 20130521, May 2014.

[22] K. Smith, H. Azami, M. A. Parra, J. Starr, and J. Escudero, 'ClusterSpan Threshold: An Unbiased Threshold for Binarising Weighted Complete Networks in Functional Connectivity Analysis', in Proc. 37th Ann. Int. Conf. IEEE Eng. Med. Biol. Soc., Milan, Italy, 2015, pp. 2840-2843.

[23] J. Escudero, E. Acar, A. Fernández, and R. Bro, 'Multiscale entropy analysis of resting-state magnetoencephalogram with tensor factorisations in Alzheimer's disease', Brain Res. Bull., vol. 119, Part B, pp. 136-144, Oct. 2015

[24] P. Holme and J. Saramäki, ‘Temporal networks', Phys. Rep., vol. 519 , no. 3, pp. $97-125$, Oct. 2012. 